Pacific Journal of Mathematics

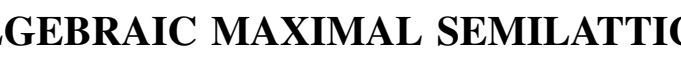




\section{ALGEBRAIC MAXIMAL SEMILATTICES}

\section{J. W. STEPP}

A topological semigroup $S$ is maximal if it is closed in each topological semigroup that contains it. The semigroup $S$ is called absolutely maximal if each continuous image is maximal. In this paper we are concerned with those discrete semilattices that are absolutely maximal. Thus we are concerned with those algebraic conditions on a semilattice which force it to be topologically closed.

In [9] Stralka studies those semigroups which have the congruence extension property. The semilattices we are concerned with and all their homomorphic images have this property. In fact, every congruence on such a semilattice $S$ is closed. Thus $S$ admits a compact Hausdorff topology $\mathscr{F}(S)$ under which multiplication is continuous. By [5] $S$ admits a unique such topology. Also, since $S$ has the congruence extension property for finite subsemilattices, the topology $\mathscr{F}(S)$ has a base which consists of subsemilattices [3].

In §II we give definitions, and we give necessary and sufficient conditions for a sublattice of a compact lattice to be closed. In §III we characterize those discrete semilattices and lattices which are absolutely maximal. Also, we show $(S, \mathscr{F}(S))$ is stable and 0dimensional. In §IV we indicate how absolutely maximal discrete semilattices are constructed from a class of simple examples.

II. Definitions. Let $S$ denote a topological semilattice. The Bohr compactification of $S$ is a pair $\left(B(S), b_{s}\right)$ where $B(S)$ is a compact semilattice, $b_{s}: S \rightarrow B(S)$ is a continuous homomorphism and if $f: S \rightarrow T$ is a continuous homomorphism with $T$ a compact semilattice, then there is a unique continuous homomorphism which makes the following diagram commute:

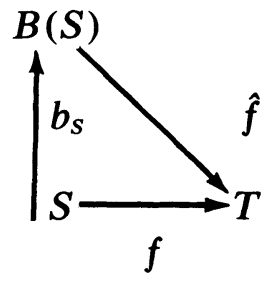

For the existence of the Bohr Compactification see either [1] or [2]. 
For each $U \subseteq S$ with $U \neq \emptyset$ let $M(U)=\{y \in S \mid$ there is an $x \in U$ with $x y=x\}, L(U)=U \cdot S$ and $C L(U)$ denotes the closure of $U$. Define $\leqq$ on $S$ by $x \leqq y$ if and only if $x y=x$. Let $\left\{x_{a}\right\}_{a \in A}$ be a net in $S$. To say $x_{\alpha} \uparrow x$ means the net converges to $x$ and $x_{\alpha} \leqq x_{\beta}$ whenever $\alpha \leqq \beta$. We define $x_{\alpha} \downarrow x$ is a similar manner. For a topological semilattice $T \operatorname{Hom}(S, T)$ denotes the collection of continuous homomorphisms from $S$ to $T$. Let $I$ denote the unit interval with $x y=\min \{x, y\}$ and let $I_{1}=\{0,1\} \subseteq I$.

Proposition 1. Let $L$ be a compact topological semilattice with identity element and let $A$ be a sublattice of $S$. Then $A$ is closed if and only if $A$ is complete.

Proof. Assume $A$ is complete and let $x \in \operatorname{CL}(A)$. Let $\mathcal{U}$ be the collection of sequences of open sets about $x$ having the following property; $\left\{U_{n}\right\}_{n=1}^{\infty} \in \mathcal{U}$ if and only if $\operatorname{CL}\left(U_{n+1}\right) \wedge \operatorname{CL}\left(U_{n+1}\right) \subseteq U_{n}$ for $n=1,2, \cdots$. Partially order $\mathcal{U}$ by $\left\{U_{n}\right\}_{n=1}^{\infty} \leqq\left\{V_{n}\right\}_{n=1}^{\infty}$ if $V_{n} \subseteq U_{n}$ for all $n$. Then $\mathcal{U}$ with this partial order is a directed set. Now fix $\alpha=$ $\left\{U_{n}\right\}_{n=1}^{\infty} \in \mathcal{U}$. Note that $\cap_{i=1}^{\infty} U_{n}=\cap_{i=1}^{\infty} \mathrm{CL}\left(U_{n}\right)$ is a sublattice of $L$ and if $\left(\cap_{i=1}^{\infty} U_{n}\right) \cap A \neq \emptyset$, then $\left(\cap_{i=1}^{\infty} U_{n}\right) \cap A$ is closed under taking inf $s$ and thus has a zero which will be denoted by $z(\alpha)$. Thus we show this intersection exists.

For each $n$ let $b_{n} \in U_{n} \cap A$ and let $\left\{a_{p}^{n}\right\}_{p=1}^{\infty}$ be the sequence given by $a_{p}^{n}=\wedge_{j=1}^{p} b_{n+j}$. Then $\left\{a_{p}^{n}\right\}_{p=1}^{\infty} \subseteq U_{n}$ and is a decreasing sequence and thus has a limit point $t$ in $\operatorname{CL}\left(U_{n}\right) \cap A$. Clearly, $t \in \operatorname{CL}\left(U_{m}\right)$ for all $m>n$ and thus $\left(\cap_{i=1}^{\infty} U_{n}\right) \cap A \neq \varnothing$. It is clear that if $\alpha, \beta \in U$ with $\alpha<\beta$, then $z(\alpha) \leqq z(\beta)$. Thus $\{z(\alpha)\}_{\alpha \in \mathscr{U}}$ is an increasing net in $A$ which converges to $x$. Since $A$ is complete, and $l$ compact, $x \in \operatorname{CL}(A)$.

In [5] Lawson defines $B^{+}$for an ideal in a semilattice $S$ to be $\{x \mid$ there is a net $\left\{x_{\alpha}\right\}_{\alpha \in \Gamma} \subseteq B$ with $\left.x_{\alpha} \uparrow x\right\}$. He shows for an ideal $B$ in a compact semilattice $S$ is closed if and only if $B^{+}=B$. Thus one has

Proposition 2. Let $B$ be a subsemilattice of a compact semilattice $S$. Then $B$ is closed if and only if $B$ contains arbitrary infs and $B=B^{+}$.

We also need the following from [5].

PROPOSITION 3. Let $S$ and $T$ be compact semilattices and lef $f$ be a homomorphism from $S$ to $T$. Then $f$ is continuous if and only if $f$ has the property that $f\left(x_{\alpha}\right) \uparrow f(x)$ whenever $x_{\alpha} \uparrow x$ and $f\left(y_{\alpha}\right) \downarrow f(y)$ whenever $y_{\alpha} \downarrow y$.

Comment 4. It is not the case that a complete lattice necessarily admits a compact Hausdorff topology for which both operations are 
continuous. For consider the lattice on the integers with 0 the smallest element, 1 the largest element and each maximal chain having three elements. However, $(Z, \wedge)$ does admit a compact Hausdorff topology with $\wedge$ continuous.

III. Maximal semilattices and lattices. Throughout this section $S$ will denote a discrete semilattice which is absolutely maximal. Since $S$ is a locally compact semilattice with a base for the topology which consist of subsemilattices, $\operatorname{Hom}(S, I)$ separates points [4]. Thus there exists a continuous injection $\alpha$ from $S$ into a compact semilattice. Since $\alpha(S)$ is closed it is compact and $S$ therefore admits a compact topology $\mathscr{F}(S)$ with multiplication continuous. By [5], $\mathscr{F}(S)$ is unique, and therefore $(\alpha(S), \alpha)$ is the Bohr compactification of $S$. Note that $\alpha(S)$ is the Bohr compactification of $\alpha(S)$ with the discrete topology. Therefore, we first characterize those compact semilattices $T$ which are the Bohr compactification of $T$ with the discrete topology.

For a semilattice $T$ we let $T_{d}$ denote $T$ with the discrete topology.

PROPOSITION 5. Let $T$ be a compact semilattice with $T=$ $B\left(T_{d}\right)$. Then

(a) $\operatorname{Hom}\left(T, I_{1}\right)$ separates points.

(b) If $U$ is a subsemilattice of $T$, then $M(U)$ is both open and closed.

(b') Each prime ideal of $T$ is both open closed

(c) $\operatorname{dim} S=0$.

Proof. (a) Let $x, y \in T$ and assume $x \notin M(y)$. Let $\phi: T \rightarrow I_{1}$ be given by $\phi(s)=1$ if $s \in M(y)$ and 0 otherwise. Since $T=B\left(T_{d}\right)$, $\phi \in \operatorname{Hom}\left(T, I_{1}\right)$, and $\phi(y)=1 \neq 0=\phi(x)$. It now follows that Hom $\left(T, I_{1}\right)$ separates points.

(b), (b') Same as (a).

(c) Since $\operatorname{Hom}\left(S, I_{1}\right)$ separates points and $S$ is compact, $S$ can be embedded in a $\mathbf{0}$-dimensional semilattice and is therefore $\mathbf{0}$-dimensional.

LEMMA 6. Let $T$ be a compact semilattice with $M(U)$ both open and closed for each subsemilattice $U$ of $S$. If $C$ is a chain in $T$, then $C$ is finite.

Proof. Assume $T$ has an infinite chain $C$. Then $\mathrm{CL}(C)$ is a chain and must have a limit point $z$. Since $M(z)$ is open, there is net $\left\{x_{\alpha}\right\}_{\alpha \in \Gamma}$ in $C$ with $x_{\alpha} \downarrow z$ and $x_{\alpha} \neq z$ for each $\alpha \in \Gamma$. Let $N=\cap_{\alpha \in \Gamma} M(x \alpha)$; then 
$M(U)$ is closed with $z \notin M(U)$ with is a contradiction. Thus $C$ must be finite.

Proposition 7. Let T be a compact semilattice. Then the following are equivalent :

(a) $T=B\left(T_{d}\right)$.

(b) $M(U)$ is both open and closed for each subsemilattice $U$ of $S$.

(c) Each chain in $T$ is finite.

(d) $\operatorname{Hom}\left(T, I_{1}\right)=\operatorname{Hom}\left(T_{d}, I_{1}\right)$.

(e) There is a compact semilattice $R$ with $|R|>1$ with $\operatorname{Hom}(T, R)=\operatorname{Hom}\left(T_{d}, R\right)$.

Proof. (a) $\Rightarrow$ (b) $\Rightarrow$ (e) trivial, (e) $\Rightarrow$ (b) by proof Proposition 5, (b) $\Rightarrow$ (c) by Lemma 6. Thus we show (c) $\Rightarrow$ (a).

Let $f \in \operatorname{Hom}\left(T_{d}, R\right)$ where $R$ is a compact semilattice and each chain in $T$ is finite. Let $\left\{x_{\alpha}\right\}_{\alpha \in \Gamma}$ be a net in $T$ with $x_{\alpha} \downarrow x$. Since each chain is finite, eventually $x_{\alpha}=x$ and $f\left(x_{\alpha}\right) \downarrow f(x)$. Similarly, if $y_{\alpha} \uparrow y$ then $f\left(y_{\alpha}\right) \uparrow f(y)$. By Proposition $4, f \in \operatorname{Hom}(T, R)$ and thus $T=$ $B\left(T_{d}\right)$.

Lemma 8. Let $T$ be a topological semilattice and let $R$ be $a$ subsemilattice of $T$ with each chain finite. Then $R$ is closed.

Proof. Let $x \in \mathrm{CL}(R)$. Let $\mathcal{U}$ be the collection of sequences of open sets about $x$ satisfying; $\left\{U_{n}\right\}_{n=1}^{\infty} \in \mathcal{U}$ if and only if $U_{n+i} U_{n+1} \leqq U_{n}$ for all $n$. Partially order $\mathcal{U}$ by $\left\{U_{n}\right\}_{n=1}^{\infty} \leqq\left\{V_{n}\right\}_{n=1}^{\infty}$ if and only if $V_{n} \subseteq U_{n}$ for all $n$. Then $U$ with this partial order is a directed set. Fix $\left\{U_{n}\right\}_{n=1}^{\infty}=\alpha \in \mathcal{U}$. Then $\cap_{i=1}^{\infty} U_{n}$ is a subsemilattice of $T$ and if ( $\cap$ $\left.{ }_{i=1}^{\infty} U_{n}\right) \cap R \neq \emptyset$, then $\left(\cap_{i=1}^{\infty} U_{n}\right) \cap R$ has a zero. For each positive integer $n$ let $b_{n} \in U_{n} \cap R$, and for each positive integer $p$ let $a_{p}^{n}=$ $b_{n+1} b_{n+2} \cdots b_{n+p}$. As before, $a_{p}^{n} \in U_{n}$ for all $p$. Since each chain in $R$ is finite, there is a $q$ such that if $p>q$ then $a_{q}^{n}=a_{q}^{n}$. Thus $\left\{a_{p}^{n}\right\}_{p=1}^{\infty}$ converges to $a^{n}$ in $U_{n}$. Clearly, if $m>n$ then $a^{m} \geqq a^{n}$. Thus there is a $m_{0}$ such that if $n \geqq m_{0}$ then $a^{n}=a^{m_{0}}$. It now follows that $a^{m_{0}} \in\left(\cap_{i=1}^{\infty} U_{n}\right) \cap R$. Let $z(\alpha)$ be the zero of $\left(\cap_{i=1}^{\infty} U_{n}\right) \cap R$. Thus $\{z(\alpha)\}_{\alpha \in \mathscr{U}}$ converges to $x$. Thus $r=x \in R$ and $R$ is closed.

We now summarize our results in the form of a theorem.

TheOREM 9. Let $T$ be a discrete semilattice. Then $T$ is absolutely maximal if and only if each maximal chain in $T$ is finite.

It is clear that we also have 
COROllary 10. Let $L$ be a discrete lattice with each chain finite. Then each lattice homomorphic image of $L$ is closed.

We close this section with some additional properties a semilattice $T$ with $T=B\left(T_{d}\right)$ must have. The proofs are all straightforward and will be omitted.

Proposition 11. Let $T$ be a compact semilattice with $T=$ $B\left(T_{d}\right)$. Then

(a) Each semilattice of $T$ is closed.

(b) If $R$ is a sublattice of $T$, then $R=B\left(R_{d}\right)$.

(c) If $R$ is a homomorphic image of $T$, then $R=B\left(R_{d}\right)$.

(d) $T$ is stable (that is, there are no dimension raising homomorphisms on $T$ ).

IV. Examples. Throughout this section $S_{d}$ is assumed to be a discrete absolutely maximal semilattice and $S$ will denote $B\left(S_{d}\right)$. For each $x \in S_{d}$ let $A(x)=\left\{y \in S_{d} \mid x<y\right.$ and $M(x) \cap L(y)=\{x, y\}$.

Lemma 12. For each $x \in S_{d} A(x)$ is infinite if and only if $x$ is a limit point of $S$. Further, if $A(x)$ is infinite, then $\mathrm{CL}_{S}(A(x))=$ $A(x) \cup\{x\}$.

Proof. Assume $A(x)$ is infinite and let $\left\{y_{\alpha}\right\}_{\alpha \in \Gamma}$ be a net in $A(x)$ which converges (in $S$ ) to $y$. Assume each $y_{\alpha} \neq y$. Let $z \in A(x)$; then $z y_{\alpha}=x$ if $y_{\alpha} \neq z$. Thus $z y=x$. It now follows that $y=y^{2}=\lim y y_{\alpha}=$ $\lim x=x$. Thus $\mathrm{CL}_{S}(A(x))=A(x) \cup\{x\}$ and $x$ is a limit point of $S$.

Now assume $x$ is a limit point of $S$ and let $\left\{z_{\alpha}\right\}_{\alpha \in \Gamma}$ be a net in $S$ which converges to $x$ and $z_{\alpha} \neq x$. For each $\alpha \in \Gamma$ let $x_{\alpha} \in A(x) \cap$ $L\left(Z_{\alpha}\right)$. Such $x_{\alpha}$ 's exist since each chain in $T$ is finite. Thus $\left\{x_{\alpha}\right\}_{\alpha \in \Gamma}$ is a net in $A(x)$ which converges to $x$. It now follows that $A(x)$ is infinite.

EXAMPLE 13. Let $X$ be a compact well-ordered space and let $B$ be the set of limit points of $X$. Let $\rho$ be defined on $X$ by $x \rho y$ if and only if $x=y$ or $x, y \in B$. Then $X / \rho$ is a compact Hausdorff space. Define multiplication on $X / \rho$ by $[x][y]=[x]$ if $[x]=[y]$ and $B$ otherwise. Then $X / \rho$ with this multiplication is a compact semilattice with each chain finite. Thus $(X / \rho)_{d}$ is an absolutely maximal semilattice. 
EXAMPLE 14. Let $T=\{((1 / n),(1 / p)) \mid n, p$ positive integers, $n \leqq$ $p \leqq 2 n\} \cup\{(0,0)\}$ with multiplication defined by

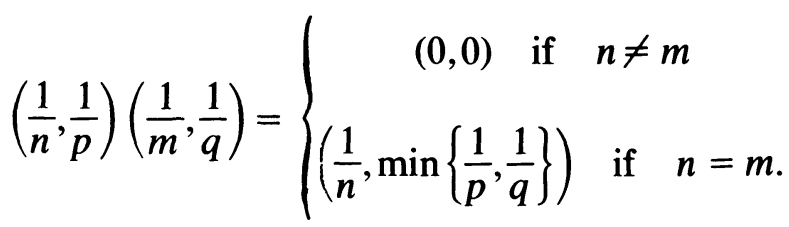

Then each chain in $T_{d}$ is finite and thus $T_{d}$ is absolutely maximal. Note that although chain in $T$ is finite there is no upper bound on the length of chains.

Observation 15. Let $x$ be a limit point of $S$. Then $\mathrm{CL}_{S}(A(x))$ is isomorphic to $X / \rho$ for a suitable compact well-ordered space $X$ (see example 13).

Observation 16. There is a discrete semilattice $T_{d}$ which is absolutely maximal and the set of limit points of $T$ is $S$.

Question 17. If $S$ is a maximal semilattice is it absolutely maximal?

Question 18. Are these reasonable conditions one can impose on a locally compact semilattice to guarantee that it be maximal?

\section{REFERENCES}

1. J. F. Buglund and K. H. Hofmann, Compact semitopological semigroups and weakly almost periodic functions, Lecture Notes; Springer-Verlag, New York (1967).

2. M. Friedberg and J. W. Stepp, A note on the Bohr Compactification, Semigroup Forum, 6 (1973), 362-364.

3. A.Y.W. Lau, Small semilattices and costability, Doctoral Dissertation, The University of Houston, Houston, Texas 1971.

4. J. D. Lawson, Topological semilattices with small semilattices, J. London Math. Soc., (2), (1969), 719-724.

5. - Intrinsic topologies in topological lattices and semi-lattices, (to appear).

6. J. W. Stepp, A note on maximal locally compact semigroups, Proc. Amer. Math. Soc., (20), 1 (1969), 251-253.

7. - On the free compact lattice generated by a topological semilattice, (to appear).

8. A. R. Stralka, The congruence extension property for compact topological lattices, Pacific J. Math., (38), 3 (1971), 795-802.

9. - Extending congruences on semigroups, Trans. Amer. Math. Soc., 166 (1972), 147-161.

Received February 11, 1974.

UNVERSITY OF HOUSTON 


\section{CONTENTS}

Zvi Artstein and John A. Burns, Integration of compact set-valued functions

J. A. Beachy and W. D. Blair, Rings whose faithful left ideals are cofaithful

Mark Benard, Characters and Schur indices of the unitary reflection group $[321]^{3}$

H. L. Bentley and B. J. Taylor, Wallman rings ............................. 15

E. Berman, Matrix rings over polynomial identity rings II ............... 37

Simeon M. Berman, A new characterization of characteristic functions of absolutely continuous distributions ........................ 323

Monte B. Boisen, Jr. and Philip B. Sheldon, Pre-Prüfer rings ............ 331

A. K. Boyle and K. R. Goodearl, Rings over which certain modules are injective

J. L. Brenner, R. M. Crabwell and J. Riddell, Covering theorems for finite nonabelian simple groups. $V$

H. H. Brungs, Three questions on duo rings .................................... 345

Iracema M. Bund, Birnbaum-Orlicz spaces of functions on groups ....351

John D. Elwin and Donald R. Short, Branched immersions between 2-manifolds of higher topological type

J. K. Finch, The single valued extension property on a Banach space ................................................................................. 61

J. R. Fisher, A Goldie theorem for differentiably prime rings ............ 71

Eric M. Friedlander, Extension functions for rank 2, torsion free abelian groups ...................................................................... 371

J. Froemke and R. Quackenbusch, The spectrum of an equational class of groupoids

B. J. Gardner, Radicals of supplementary semilattice sums of

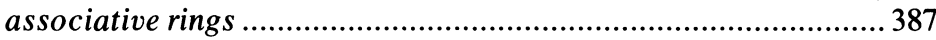

Shmuel Glasner, Relatively invariant measures ...............................393

G. R. Gordh, Jr. and Sibe Mardešić, Characterizing local connectedness in inverse limits...

S. Graf, On the existence of strong liftings in second countable topological spaces

S. Gudder and D. Strawther, Orthogonally additive and orthogonally increasing functions on vector spaces ........................................427

F. Hansen, On one-sided prime ideals .......................................... 79

D. J. Hartfiel and C. J. Maxson, A characterization of the maximal monoids and maximal groups in $\beta x$.

Robert E. Hartwig and S. Brent Morris, The universal flip matrix and the generalized faro-shuffle 


\section{Pacific Journal of Mathematics}

Vol. 58, No. 1

March, 1975

John Allen Beachy and William David Blair, Rings whose faithful left ideals are cofaithful .................................... 1

Herschel Lamar Bentley and Barbara June Taylor, Wallman rings ........ 15

Elizabeth Berman, Matrix rings over polynomial identity rings. II ...... 37

Ann K. Boyle and Kenneth R. Goodearl, Rings over which certain modules are injective ................................. 43

J. L. Brenner, Robert Myrl Cranwell and James Riddell, Covering theorems for finite nonabelian simple groups. $V \ldots \ldots \ldots \ldots \ldots \ldots \ldots \ldots \ldots$

James Kenneth Finch, The single valued extension property on a Banach

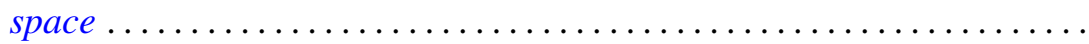

John Robert Fisher, A Goldie theorem for differentiably prime rings........ Friedhelm Hansen, On one-sided prime ideals .................... Jon Craig Helton, Product integrals and the solution of integral equations..........................................

Barry E. Johnson and James Patrick Williams, The range of a normal

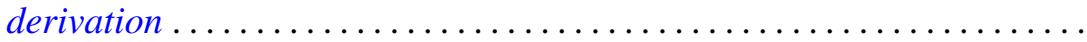

Kurt Kreith, A dynamical criterion for conjugate points ............ 123

Robert Allen McCoy, Baire spaces and hyperspaces .............. 133

John McDonald, Isometries of the disk algebra ................ 143

H. Minc, Doubly stochastic matrices with minimal permanents ......... 155

Shahbaz Noorvash, Covering the vertices of a graph by vertex-disjoint paths. ...

Theodore Windle Palmer, Jordan *-homomorphisms between reduced Banach*-algebras

Donald Steven Passman, On the semisimplicity of group rings of some

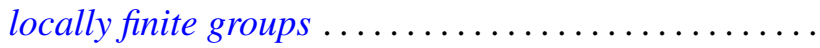

Mario Petrich, Varieties of orthodox bands of groups .

Robert Horace Redfield, The generalized interval topology on distributive

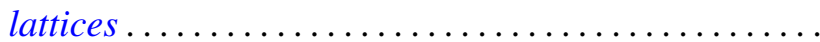

James Wilson Stepp, Algebraic maximal semilattices .... . .

Patrick Noble Stewart, A sheaf theoretic representation of rings with Boolean orthogonalities ........................

Ting-On To and Kai Wing Yip, A generalized Jensen's inequality......... 255

Arnold Lewis Villone, Second order differential operators with self-adjoint

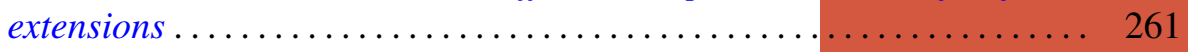

Martin E. Walter, On the structure of the Fourier-Stieltjes algebra ....... 267

John Wermer, Subharmonicity and hulls .................... 283

Edythe Parker Woodruff, A map of $E^{3}$ onto $E^{3}$ taking no disk onto a

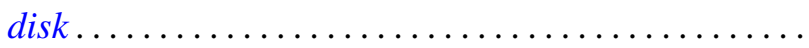

